\title{
The personal side to genetic testing
}

\section{Jennifer Shuldiner}

University of Toronto

Genetic testing for cancer susceptibility is a premier example of personalized medicine (1). Owing to the development of next-generation sequencing, genetic testing is rapidly evolving from phenotype-driven sequential testing to multiplex testing. This new process has significantly reduced cost and increased the efficiency of gene sequencing (2). Multiplex testing uses whole genome sequencing to generate information on predefined target genes. Genetic cancer panels are an example of how multiplex testing can be applied clinically to offer individual risk information (3). Panel-based testing groups similar genes either by cancer type or level of associated cancer risk. This grouping technique reduces the potential for information overload, while still providing sufficient information for meaningful informed consent (4). With the growing number of identified cancer susceptibility genes, along with potential time and cost savings of multiplex testing, it is anticipated that panel-based testing will be incorporated into routine clinical care in the near future. While the clinical application of this form of testing should be guided in part by medical outcomes such as assessment of mortality and morbidity, it should also include broader outcomes including personal utility, which comprises effects that are personal, psychological, and social.

Panel-based genetic testing increases the likelihood of detecting an affected individual's disease-causing mutation and provides a more complete genetic evaluation (3). However, there are risks and limitations, as some genes have not yet been sufficiently studied, leaving their cancer risk unclear. There are also moderate-penetrance mutations, such as CHEK2, for which the optimal management protocols regarding screening and cancer prevention are unknown (5). Finally, research to provide risk estimates for individuals who may have multiple moderate-penetrance mutations is lacking. Therefore, test findings can be confusing and anxiety-provoking for patients, without apparent benefit. Providers need direction on how to guide individuals who receive genetic re- sults in the absence of clear risk approximation, when there are incidental findings and optimal management is unknown.

Any new test, drug, or procedure typically undergoes a comprehensive assessment, including an evaluation of analytic validity (e.g. characteristic of the test; precision and reliability of the measurement of the assay), clinical validity (e.g. the ability of a test to predict the trait or condition in question), and clinical utility (e.g. improved measurable medical outcomes). However, small sample sizes and low incidence rates of many hereditary cancers have resulted in limited research demonstrating improved survival or health outcomes as a consequence of interventions informed by a genetic predisposition. For example, research on age-specific risk for many genes is inadequate. Consequently, recommendations regarding preventive measures are uncertain.

In light of limited evidence for clinical utility, some have argued that the evaluation of benefits of panelbased testing in clinical practice should incorporate personal utility $(6,7)$. Personal utility is defined as genomic information that informs and guides personal understanding, decision-making, and behavioural responses to genetic test results (8). Access to genetic information can increase an individual's sense of control, allowing them to advocate for health-related decisions (6), reinforce adherence to clinical recommendations, and inform reproductive decisions (7). Personal utility can include important outcomes, such as greater compliance with screening recommendations or the initiation of risk-reducing behaviours. Personal utility can also include emotional effects (e.g. comfort or distress), collective effects (e.g. impacting family dynamics or stigmatization), and cognitive effects (e.g. increased disease comprehension). For example, individuals tested for Huntington's disease, a life-limiting illness with no preventative or therapeutic options, may experience personal utility from such a test through the conferred psychological, social, and practical benefits $(7,9)$. 
Personal utility is an important concept to consider when discussing the advantages and drawbacks of genetic testing. However, the subjective and multidimensional nature of this construct presents challenges in terms of measurement. One study on panel-based genetic testing for breast cancer susceptibility measured personal utility with a 12-point scale and found that perceived utility was modest after pre-test counselling (4). However, there was a significant increase in perceived utility after testing, which was highest among those receiving a positive genetic test result (4). This type of study underscores the importance of exploring the construct of personal utility in genetic testing, especially for those tests with uncertain clinical utility due to limited research.

When considering the clinical relevance of panelbased testing, personal utility is a criterion that should be weighed in the context of clinical utility, validity, cost-effectiveness, and health service delivery (10). There is a lack of recognition of the significance of this outcome, and empirical data is required to elucidate this concept. Panel-based genetic testing is a practical form of genomics and its full benefit will be undermined if we ignore the impact on the person.

\section{References}

1. Pasche B, Absher D. Whole-genome sequencing: a step closer to personalized medicine. JAMA. 2011;305(15):1596-1597.

2. Walsh T, Lee MK, Casadei S, Thornton AM, Stray SM, Pennil C, et al. Detection of inherited mutations for breast and ovarian cancer using genomic capture and massively parallel sequencing. Proc Natl Acad Sci USA. 2010;107(28):12629-12633.

3. Tung N, Battelli C, Allen B, Kaldate R, Bhatnagar S, Bowles K, et al. Frequency of mutations in individuals with breast cancer referred for BRCA1 and BRCA2 testing using next-generation sequencing with a 25-gene panel. Cancer. 2015;121(1):25-33.

4. Bradbury AR, Patrick-Miller $\amalg$, Egleston BL, DiGiovanni L, Brower J, Harris D, et al. Patient feedback and early outcome data with a novel tiered-binned model for multiplex breast cancer susceptibility testing. Genet Med. 2015;18(1):25-33.

5. Robson M. CHEK2, breast cancer, and the understanding of clinical utility. Clin Genet. 2010;78(1):8-10.
6. Foster MW, Sharp RR. The contractual genome: How direct-toconsumer genomic services may help patients take ownership of their DNA. Per Med. 2008;5(4):399-404.

7. Grosse SD, Khoury MJ. What is the clinical utility of genetic testing? Genet Med. 2006;8:448-450.

8. Bunnik EM, Janssens ACJW, Schermer MHN. Personal utility in genomic testing: is there such a thing? J Med Ethics. 2015;41(4):322-326.

9. Haddow JE, Palomaki GE. ACCE: A model process for evaluating data on emerging genetic tests. In: Khoury MJ, Little J, Burke W. Human Genome Epidemiology: A Scientific Foundation for Using Genetic Information to Improve Health and Disease. USA: Oxford University Press; 2004:217-233.

10. Bombard $\mathrm{Y}$, Bach PB, Offit $\mathrm{K}$. Translating genomics in cancer care. J Natl Compr Canc Netw. 2013;11(11):1343-1353.

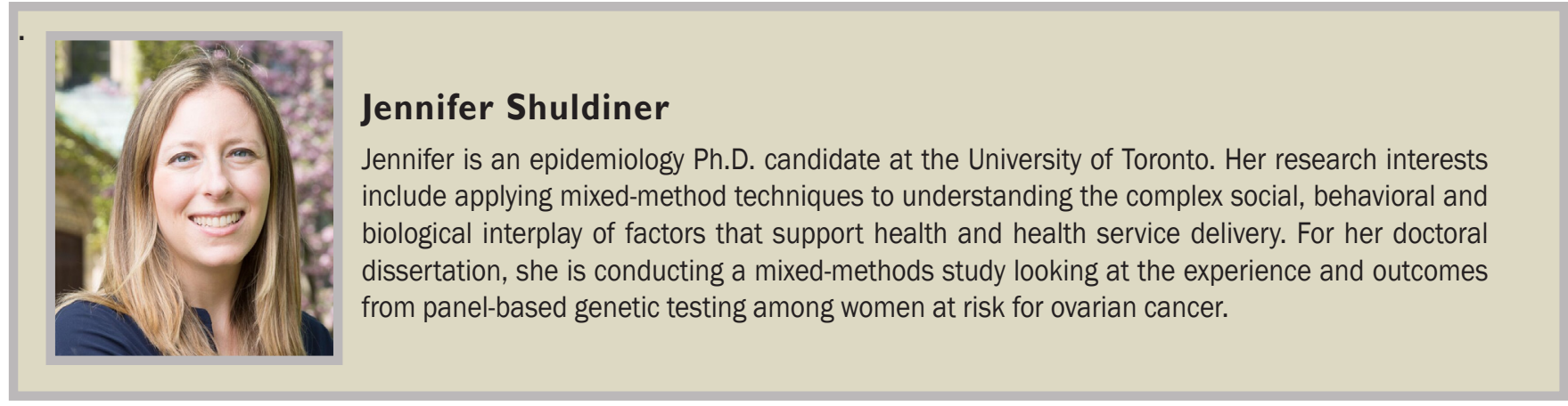

